\title{
CRIg-expressing peritoneal macrophages are associated with disease severity in patients with cirrhosis and ascites
}

Katharine M. Irvine, ${ }^{1}$ Xuan Banh, ${ }^{1}$ Victoria L. Gadd, ${ }^{1}$ Kyle K. Wojcik, ${ }^{1}$ Juliana K. Ariffin, ${ }^{2,3}$ Sara Jose, ${ }^{1}$ Samuel Lukowski, ${ }^{2}$ Gregory J. Baillie, ${ }^{2}$ Matthew J. Sweet, ${ }^{2,3}$ and Elizabeth E. Powell1,4

${ }^{1}$ School of Medicine, ${ }^{2}$ Institute for Molecular Bioscience (IMB), and ${ }^{3}$ IMB Centre for Inflammation and Disease Research, The University of Queensland, Brisbane, Queensland, Australia. ${ }^{4}$ Department of Gastroenterology and Hepatology, Princess Alexandra Hospital, Brisbane, Queensland, Australia.

Infections are an important cause of morbidity and mortality in patients with decompensated cirrhosis and ascites. Hypothesizing that innate immune dysfunction contributes to susceptibility to infection, we assessed ascitic fluid macrophage phenotype and function. The expression of complement receptor of the immunoglobulin superfamily (CRIg) and CCR2 defined two phenotypically and functionally distinct peritoneal macrophage subpopulations. The proportion of CRIgh macrophages differed between patients and in the same patient over time, and a high proportion of CRIg ${ }^{\text {hi }}$ macrophages was associated with reduced disease severity (model for endstage liver disease) score. As compared with CRIg ${ }^{10}$ macrophages, CRIghi macrophages were highly phagocytic and displayed enhanced antimicrobial effector activity. Transcriptional profiling by RNA sequencing and comparison with human macrophage and murine peritoneal macrophage expression signatures highlighted similarities among CRIghi cells, human macrophages, and mouse $\mathrm{F} 4 / 80^{\text {hi }}$ resident peritoneal macrophages and among CRIg ${ }^{\text {lo }}$ macrophages, human monocytes, and mouse $\mathrm{F} 4 / 80^{10}$ monocyte-derived peritoneal macrophages. These data suggest that CRIghi and CRIg ${ }^{\text {lo }}$ macrophages may represent a tissue-resident population and a monocytederived population, respectively. In conclusion, ascites fluid macrophage subset distribution and phagocytic capacity is highly variable among patients with chronic liver disease. Regulating the numbers and/or functions of these macrophage populations could provide therapeutic opportunities in cirrhotic patients.

Conflict of interest: The authors have declared that no conflict of interest exists.

Submitted: February 4, 2016 Accepted: April 21, 2016 Published: June 2, 2016

Reference information: JCI Insight. 2016;1(8):e86914. doi:10.1172/ji.insight.86914.

\section{Introduction}

Most of the morbidity and mortality in chronic liver disease occurs in patients with advanced fibrosis or cirrhosis. Cirrhosis typically has an asymptomatic phase (compensated cirrhosis) followed by a rapidly progressive phase (decompensated cirrhosis) signaled by the development of complications of liver dysfunction (ascites, variceal bleeding, encephalopathy, jaundice). Patients with decompensated cirrhosis have high morbidity and mortality and consequently place a heavy burden on health resources (1). Bacterial infections, which occur in one-third of admitted patients with cirrhosis, account for a 4-fold increase in mortality (2), and the high mortality rate after infection ( $28.6 \%$ at 1 month, $63 \%$ at 1 year) has not changed substantially over recent decades $(2,3)$.

Ascites, an accumulation of fluid in the peritoneal cavity, is the most frequent complication of cirrhosis. It is associated with poor prognosis, reduced quality of life, and increased hospital admissions. Ascites fluid is also the most common site of infection in patients with cirrhosis; spontaneous bacterial peritonitis (SBP) occurs in approximately $3 \%$ of outpatients and $10 \%$ of inpatients (2). Increased susceptibility of cirrhotic patients to infection is likely multifactorial, a consequence of pathological gut bacterial translocation and impaired/altered immune function (4). Although thought to be caused by gut bacterial translocation, SBP is diagnosed by ascites fluid neutrophil count, as $>60 \%$ of SBP cases are culture negative, necessitating empirical antibiotic treatment (4). Changing bacterial and resistance patterns in patients with cirrhosis have been attributed to the increasing use of antibiotic prophylaxis and invasive procedures $(5,6)$, highlighting the importance of ensuring appropriate antibiotic use and of investigating effective adjunct therapies, such 
as enhancing immune function. We previously reported that, even in the absence of overt infection and despite SBP prophylaxis, ascites bacterial DNA levels were associated with poor clinical outcomes (death, hospital readmission) (7). Bacterial DNA levels were also associated with reduced HLA-DR expression on ascites macrophages/monocytes (CD14 ${ }^{+}$cells), suggesting a reduced antigen presentation capacity (7), which may contribute to susceptibility to infection in these patients (8). The presence of macrophages in general, however, correlated with reduced ascites neutrophils and bacterial DNA, suggesting that restoring macrophage function (e.g., HLA-DR expression; ref. 9) may be a viable therapeutic strategy.

Studies in peritoneal immunity in mice indicate the presence of developmentally distinct macrophage populations. A Gata6-dependent self-renewing population with a key role in clearing apoptotic cells, coordinating adaptive immunity, and promoting mucosal immunity makes up the majority ( 95\%) of resident peritoneal macrophages. Gata6-dependent macrophages likely derive from the omentum and self-renew by proliferation, while a minor, Gata6-independent resident population probably originate from inflammatory monocytes (10-15). Studies in mice also highlight macrophage functional plasticity during peritoneal inflammation and resolution. Despite existing in the same milieu, macrophages of different origins acquire distinct phenotypes that change throughout the inflammatory response $(16,17)$, resulting in a state of "adaptive homeostasis," which lasts for months and dictates the magnitude of subsequent inflammatory responses (17). These evolving macrophage phenotypes are potentially highly relevant to peritoneal immunity in patients with chronic liver disease, as peritoneal macrophages are constantly exposed to inflammatory stimuli from translocating bacteria due to alterations in intestinal permeability. However, in contrast to mice, human peritoneal macrophage phenotype and function has not been extensively studied. We hypothesized that human counterparts of murine resident and inflammatory peritoneal macrophages may be present in ascites fluid and play different roles in infection and inflammation, thereby influencing clinical outcomes. The aims of this study were to investigate the phenotype and function of ascites fluid macrophages to gain insight into their contribution to susceptibility to infection in cirrhotic patients and the potential to manipulate them for therapeutic benefit.

\section{Results}

CRIg and CCR2 distinguish populations of human peritoneal macrophages in ascites fluid from patients with cirrhosis and ascites. We observed that a subpopulation of macrophages $\left(\mathrm{CD} 14^{+}\right.$cells) in ascites fluid from patients with cirrhosis expressed high levels of CD14 and the complement receptor for immunoglobulin (CRIg) (Figure 1, A and B), which is expressed on several tissue resident macrophage populations (18), including the major population of murine resident peritoneal macrophages $(13,19)$, and upregulated during human monocyte differentiation into macrophages (20). CRIghi cells could frequently be clearly distinguished by their high autofluorescence (Supplemental Figure 1; supplemental material available online with this article; doi:10.1172/jci.insight.86914DS1), and they did not express CCR2, a classical monocyte marker expressed on the minor resident peritoneal macrophage population in mice, which was selectively expressed on CRIgo/neg cells (Figure 1, A and C). Together, CRIg and CCR2 afforded complete separation of the macrophage populations (hereafter referred to as CRIghi and CRIg ${ }^{\text {lo }}$. The proportion of CRIghi macrophages in ascites fluid differed between patients (median 39.1\% [IQR 31.5\%] of CD14+ ascites cells, Figure 1D) and in the same patient over time (Figure 1E). A reduction in the proportion of CRIghi macrophages was observed in 3 patients at the time of cirrhosis related clinical events (SBP, hepatic encephalopathy, and death), and a low proportion of CRIghi ascites macrophages was associated with increased liver disease severity (model for end-stage liver disease [MELD] score, spearman $\mathrm{r}=-0.74, P=0.002$, Figure $1, \mathrm{E}$ and $\mathrm{F}$ ).

In addition to CRIg expression, the major population of murine resident peritoneal macrophages is characterized by their larger size and relatively high F4/80 expression and low Ccr2, Cd11b, and MHCII expression compared with the minor $\mathrm{F} 4 / 80^{\text {lo }}$ population $(10,14)$. Infiltrating inflammatory macrophages express intermediate (Int.) levels of F4/80 and variable MHCII levels and are positive for Cd11c and Cd36 $(12,21)$. In addition to lacking CCR2 expression, ascites fluid CRIghi macrophages were larger than CRIglo macrophages (Figure 2A) and exhibited higher side scatter (Figure 2B). In contrast to mouse peritoneal macrophages, CRIghi macrophages expressed the highest levels of HLA-DR (Figure 2C), and all peritoneal macrophages expressed CD11B and CD11C (Figure 2, E and F). CRIghi macrophages also expressed high levels of CD16 (Figure 2D), CD169 (SIGLEC1, Figure 2G), and CD163 (Figure 2H). CRIghi peritoneal macrophages thus share some, but not all, of the phenotypic characteristics of murine F4/80 ${ }^{\text {hi }}$ resident peritoneal macrophages. 
A

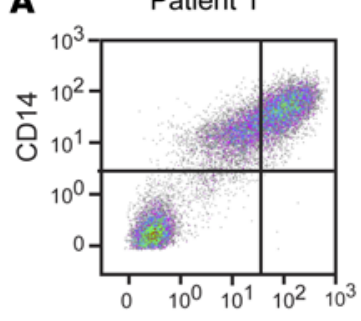

CRIg

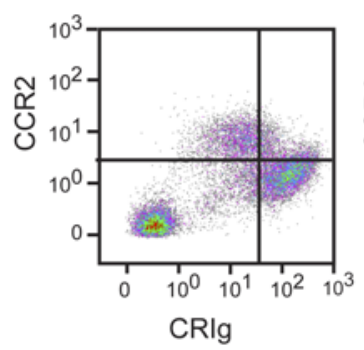

D

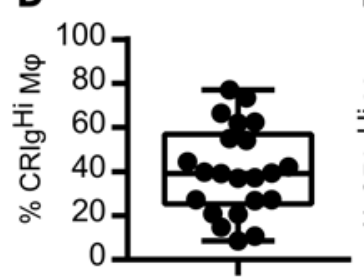

E

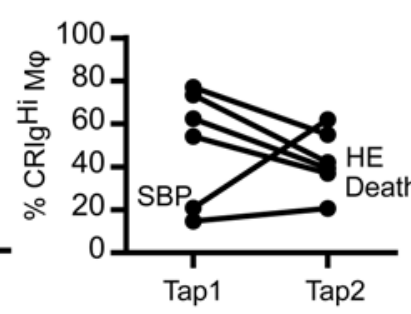

Patient 2

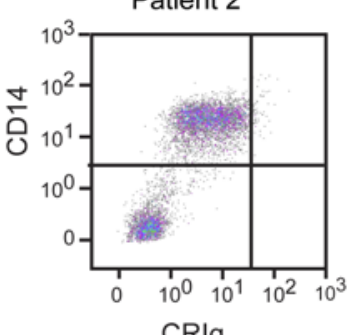

CRIg

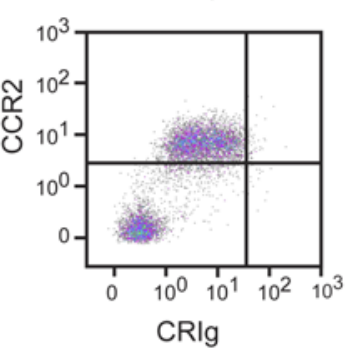

B

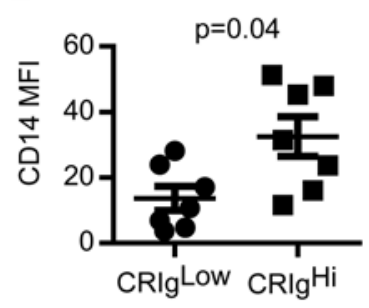

C

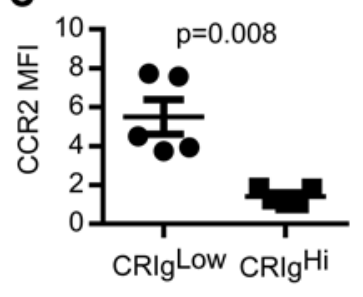

$\mathbf{F}$

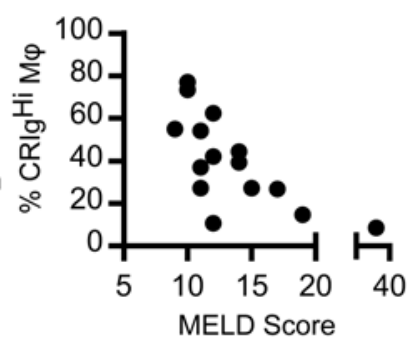

Figure 1. CRIg defines a subpopulation of macrophages in ascites fluid. (A) Representative plots showing CD14, CRIg, and CCR2 expression on cells obtained from ascites fluid from 2 cirrhotic patients. CD14-/CRIg- lymphocytes are shown for reference; however, $\mathrm{CD}^{+} 4^{+}$macrophages were gated for analysis of fluorescence intensity. (B) CD14 $(n=6)$ and (C) CCR2 $(n=5)$ median fluorescence intensity (MFI) on CRIg hi and CRIg ${ }^{10}$ macrophages. (Mann-Whitney test). (D) Proportion of CD14 ascites cells expressing CRIg in ascites fluid ( $n=22$, box represents 5 th to 95 th percentile, with whiskers indicating minimum and maximum values and the line at the median). (E) Longitudinal variation in the proportion of CD14+ ascites cells expressing CRIg. Clinically significant events are annotated ( $n=6$, approximately 3 months between paracenteses ["taps"] for each patient). SBP, spontaneous bacterial peritonitis; $\mathrm{HE}$, hepatic encephalopathy. (F) Correlation between the proportion of CD14+ ascites cells expressing CRIg and MELD score ( $n=15$, spearman $r=0.74, P=0.002$ )

CRIg macrophages exhibit increased phagocytic and microbicidal capacity. Given the role of CRIg in phagocytosis of pathogens and cellular debris, particularly complement-mediated phagocytosis (20, 22), we assessed phagocytic and microbicidal function in CRIghi and CRIg ${ }^{\text {lo }}$ macrophages. CRIghi macrophages engulfed significantly more fluorescently labeled E. coli than did CRIglo macrophages (Figure 3, A-C; $P<0.0001)$. Similarly, when infected with live $E$. coli, intracellular bacterial loads at 1 hour (largely reflecting uptake) were higher in CRIghi macrophages compared with CRIglo macrophages (Figure 3D, fresh human monocyte-derived macrophages [HMDMs] from distinct donors were infected for comparison. E. coli survival at 4 hours after infection (relative to uptake at 1 hour) was higher in CRIglo macrophages in two-thirds of experiments, suggesting a reduced microbicidal capacity compared with $\mathrm{CRIg}^{\text {hi }}$ macrophages (Figure 3E). Macrophage viability 4 hours after infection, as assessed by extracellular lactate dehydrogenase (LDH), was similar in all populations (data not shown), indicating that variation in macrophage death did not account for differences in intracellular bacterial loads. Reduced ascites fluid macrophage phagocytic capacity was associated with increased disease severity (MELD score, $\mathrm{r}_{\mathrm{s}}=-0.65$, $P=0.009$, Figure $3 F$ ). Incubation with $E$. coli bioparticles increased CRIg surface expression, which was further increased in the presence of autologous ascites fluid, and enhanced E. coli uptake (Figure 3G and data not shown; $\mathrm{r}_{\mathrm{s}}=0.87, P=0.0002, n=12$ ).

We further investigated peritoneal macrophage phagocytic capacity using fluorescent latex beads (2 $\mu \mathrm{M}$ ) opsonized with human IgG (to investigate Fc receptor-mediated phagocytosis), serum, heat-inactivated (HI) serum (to investigate complement-mediated phagocytosis), or left unopsonized. We confirmed complement C3 deposition on the latex beads after opsonization with serum but not HI serum (data not shown). Ascites macrophages were gated on CD14 and CCR2 expression (see Figure 1A), since the strong spillover fluorescence from the FitC-labeled beads into the PE channel (required for CRIg staining) could not be compensated. Ascites cells were incubated with latex beads for 1 hour in the presence or absence of $10 \mu \mathrm{g} / \mathrm{ml}$ Cytochalasin D (which blocks bead internalization by depolymerizing actin) to ascertain the relative contributions of surface-bound and internalized beads to cellular fluorescence (Figure 4, A and B). Bead binding was calculated as the percentage of bead-positive macrophages in the presence of Cytocha- 
A Forward Scatter

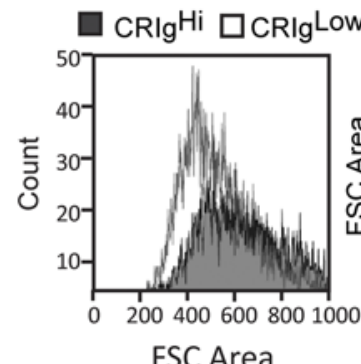

FSC Area

C HLA-DR

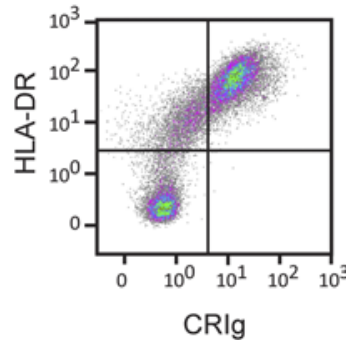

E CD11B
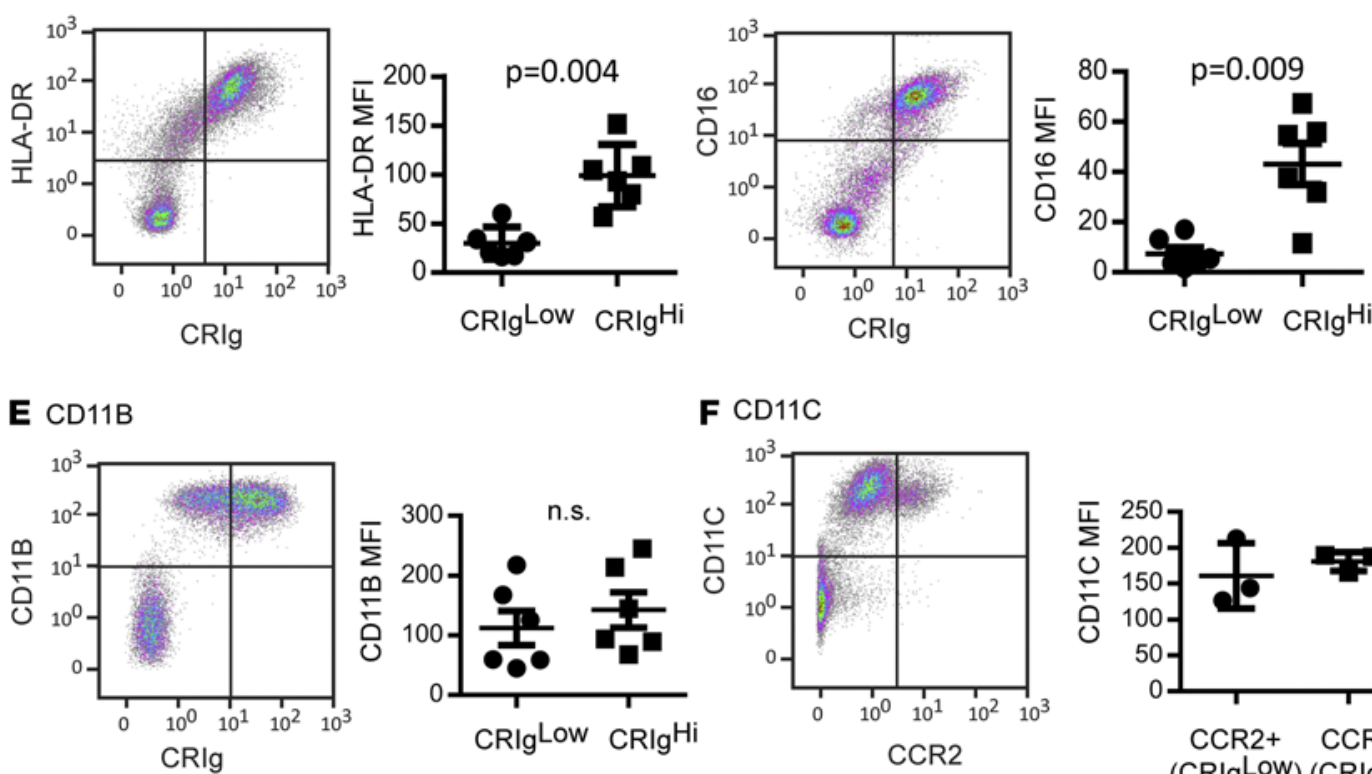

\section{F CD11C}
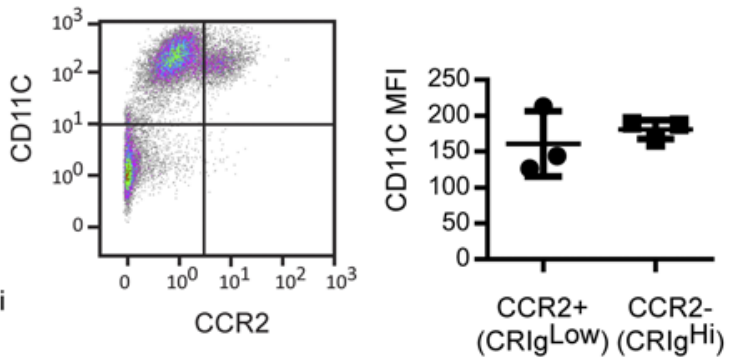

G SIGLEC1 (CD169)
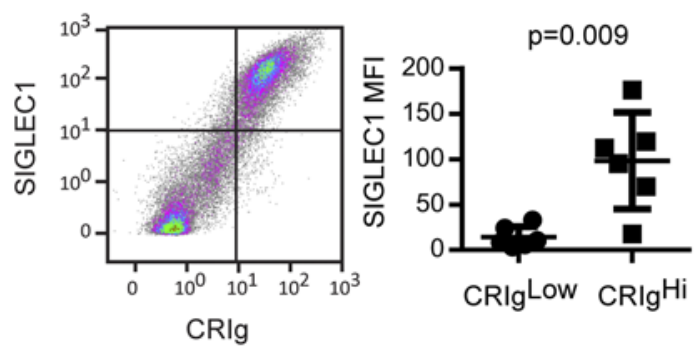

H CD163

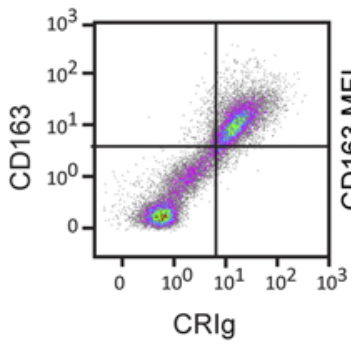

Figure 2. Phenotype of CRIghi human peritoneal macrophages. Representative (A) forward scatter (FSC) and (B) side scatter (SSC) histograms and mean area for CRIghi and CRIglo macrophages $(n=12)$. Representative dot plots showing ascites leukocyte expression of CRIg and (C) HLA-DR, (D) CD16, (E) CD11B, (F) CD11C, (C) SIGLEC1, and (H) CD163, and quantification of marker expression (median fluorescence intensity [MFI], data represent mean \pm SEM) on CRIghi and CRIglo ascites macrophages (CD14+ cells, $n=6$ except F, for which $n=3$; Mann-Whitney test). CRIg- Iymphocytes are shown for reference; however, CD14 ${ }^{+}$macrophages were gated for analysis of fluorescence intensity.

lasin D. Internalization was estimated by subtracting the proportion of macrophages that bound the latex beads from the total proportion of bead-positive macrophages in the absence of Cytochalasin $\mathrm{D}$ treatment. Opsonization primarily increased bead binding, particularly IgG, although serum increased bead binding in comparison to HI serum-opsonized and unopsonized beads, presumably due to complement deposition (Figure 4C). Unopsonized and serum-opsonized beads were efficiently internalized, whereas surface binding appeared to be greater than internalization for IgG-opsonized beads, as treatment with Cytochalasin D only modestly reduced the number of positive cells (Figure $4, \mathrm{C}$ and $\mathrm{D}$ ). CCR $2^{\mathrm{lo}} \mathrm{CRI} \mathrm{Ig}^{\mathrm{hi}}$ macrophages bound 
A No Bioparticles

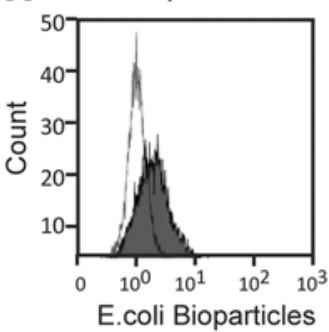

D

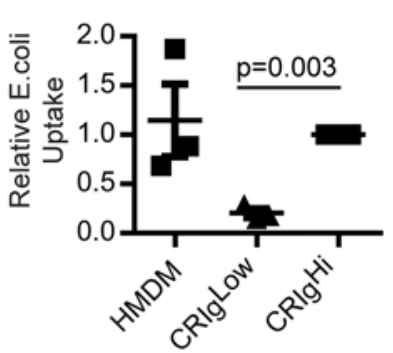

+ Bioparticles

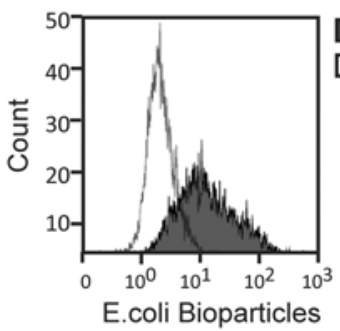

E

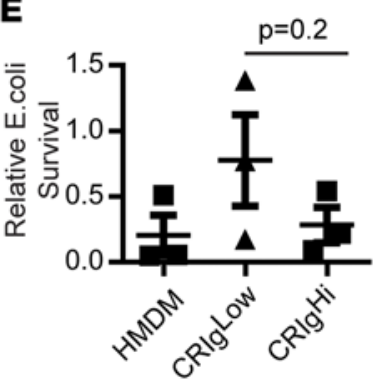

B
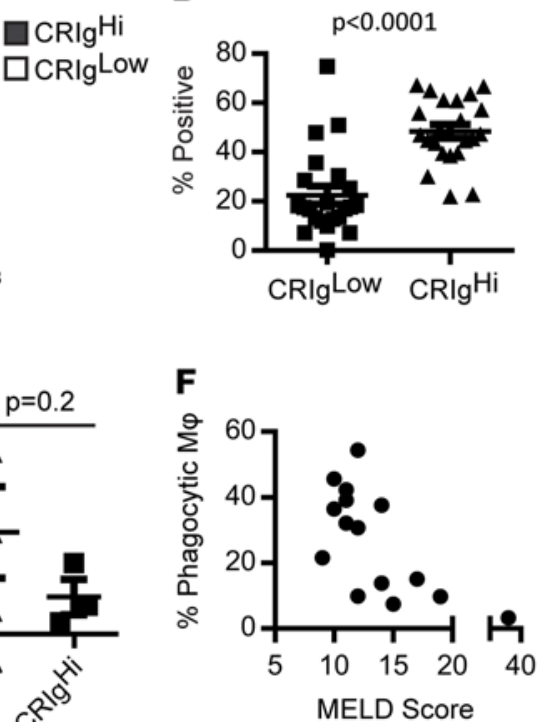

C

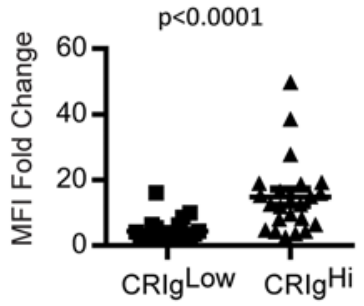

G

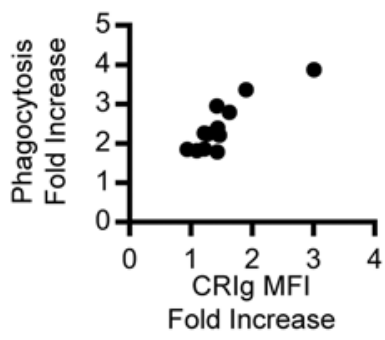

Figure 3. Increased microbial phagocytic capacity in CRIg macrophages. (A) Representative histograms showing CRIg (i) $^{\text {and }}$ CRIg ${ }^{l 0}$ fluorescence in the absence and presence of pHRodo E. coli bioparticles. (B) Proportion of CRIghi and CRIglo macrophages phagocytosing E. coli bioparticles and (C) change in median fluorescence intensity (MFI) with uptake ( $n=22$, Mann-Whitney test). (D) E. coli phagocytosis (CFU/ml 1 hour after infection, relative to CRIghi macrophages) ( $n=3,1$-sample $t$ test) and (E) killing capacity (proportion of phagocytosed $E$. coli [1 hour] surviving at 4 hours) in CRIghi $C R I g^{10}$ and human monocyte-derived macrophages (HMDM) $(n=3$, Mann-Whitney Test). Dot plot data in B-E represent mean \pm SEM. (F) Correlation between ascites fluid macrophage phagocytic capacity and MELD score $(n=15$, spearman $r=0.65, P=0.009)$. (C) Correlation between increased CRIg expression and phagocytic capacity in the presence of autologous ascites fluid (fold change compared with incubation in culture medium) $(n=12$, spearman $r=0.87, P=0.0002)$.

and internalized more of all bead types compared with CCR2 ${ }^{+} \mathrm{CRIg}{ }^{\text {lo }}$ macrophages (Figure $4, \mathrm{C}$ and D).

Transcriptomic analysis of $\mathrm{CRIg}^{+}$and CRIglo peritoneal macrophages. In order to further characterize ascites fluid macrophages, we profiled flow cytometry-sorted CRIghi and CRIglo macrophages by RNA sequencing ( $n=6$ patients). After filtering out genes with raw count $<2$ in any sample, 13,431 genes were evaluated for differential expression using the DESeq2 and EdgeR algorithms (23, 24). 2,348 genes were identified as significantly differentially expressed between the macrophage populations by both algorithms (DESeq2 adjusted $P<0.05$, EdgeR FDR $<0.05,986 \geq 2$-fold; Supplemental Table 1 and Figure 5A). mRNA expression of several macrophage markers paralleled their surface expression, with significantly higher CCR2 expression in CRIg ${ }^{\text {lo }}$ macrophages and higher expression of VSIG4 (encoding CRIg), CD163, and SIGLEC1 in CRIghi macrophages. Several transcripts expressed on other tissue macrophage populations, including FCGR1A (12) and the efferocytosis receptors MERTK and TIMD4, were also increased in CRIghi macrophages. CRIgo macrophages, by contrast, expressed $C D 36$, which is also expressed by infiltrating monocyte-derived macrophages in mice (21). Significant differences in transcript structure (exon usage) between the macrophage populations were also identified, using the DEXSeq package (25). 1,366 "exons" (counting bins; ref. 25) in 967 genes were significantly differentially used in CRIghi and CRIglo macrophages (10\% FDR, Supplemental Table 2). 23\% of differentially used exons were also differentially expressed genes, while almost $10 \%$ of differentially expressed genes exhibited significantly different exon usage between macrophage populations. Interestingly, the 14 microRNAs (MIRs) that were differentially expressed between CRIghi and CRIg ${ }^{\text {lo }}$ macrophages, including MIRs with well-characterized functions in inflammation, such as MIR21 (26), were consistently highly expressed in CRIglo cells (Figure 5B).

The Molecular Signatures Database (http://software.broadinstitute.org/gsea/msigdb/index.jsp) provides a collection of 4,782 immunologic signatures representing cell states and perturbations within the immune system defined from microarray gene expression data (collection 7, Human Immunology Project Consortium; ref. 27) for gene set enrichment analysis (GSEA). The CRIghi expression signature was most similar to the set of genes that were upregulated in monocytes following 7 days of culture (normalized enrichment score [NES] 3.26, FDR q value 0.0), supporting the view that CRIghi macrophages are a resident, or more mature, population; whereas $\mathrm{CRIg}^{\text {lo }}$ macrophages most resembled the profile of uncultured 
A

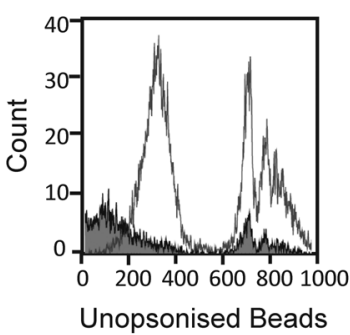

Cytochalasin D

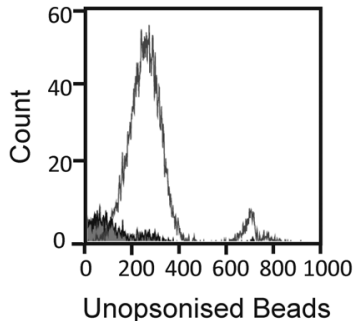

B

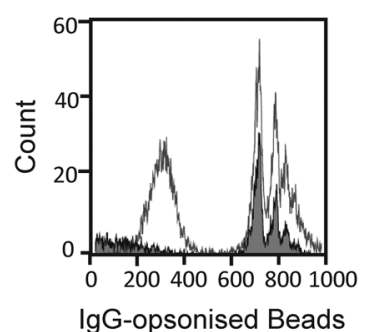

Cytochalasin D

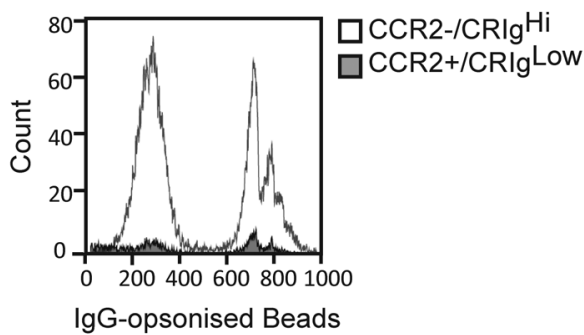

C Bead Binding

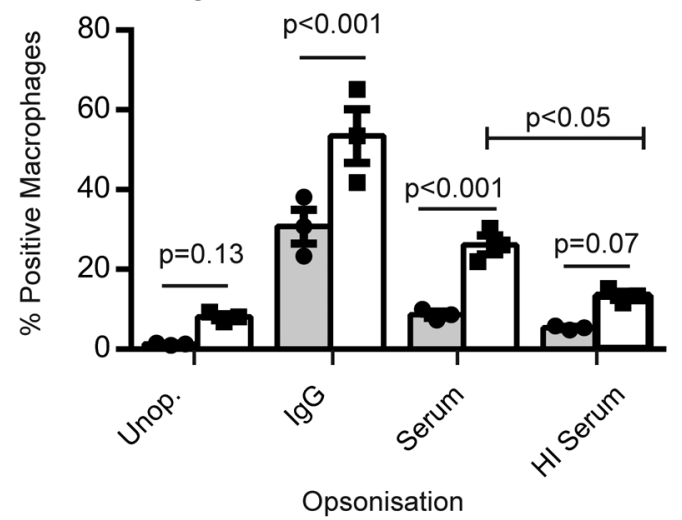

D Bead Internalisation

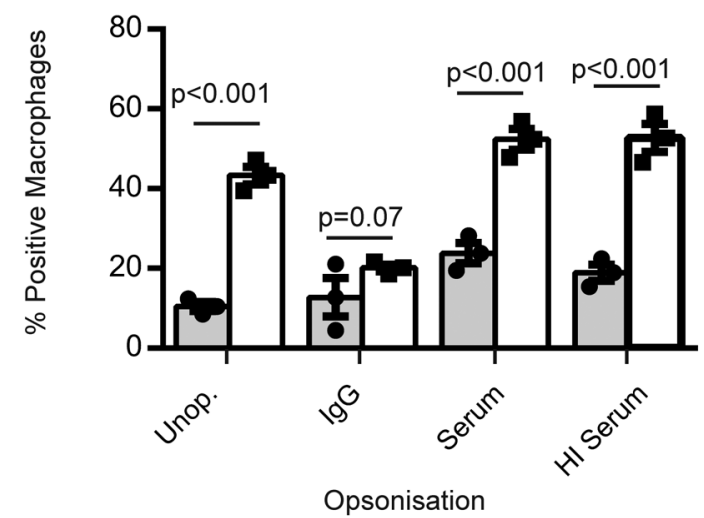

Figure 4. Increased phagocytic capacity for latex beads in CRIg macrophages. Representative histograms showing CCR2 ${ }^{\text {hi }}$ (CRIg ${ }^{\text {lo }}$ ) and CCR2- (CRIg $\left.{ }^{\text {hi }}\right)$ uptake of (A) unopsonized and (B) IgG-opsonized FitC-labeled latex beads in the absence or presence of $10 \mu \mathrm{g} / \mathrm{ml}$ Cytochalasin D. (C) Latex bead binding and (D) internalization capacity in CCR2 ${ }^{+}$(CRIg $\left.{ }^{\circ}\right)$ and CCR2- (CRIghi) macrophages ( $n=3$, multiple $t$ tests [CCR2 ${ }^{+}$vs. CCR2-] and repeated-measures 2-way ANOVA [serum vs. HI serum], data represent mean \pm SEM). HI, heat inactivated.

monocytes (NES 2.67, FDR q value 0.0) (Figure 5, C and D; ref. 28). Providing an in vivo comparison from the human peritoneum, Segura et al. recently identified and profiled a distinct population of $\mathrm{CD}_{1 \mathrm{C}^{+}}$monocyte-derived inflammatory DCs from human tumor-associated ascites that promoted Th17 differentiation (29) and published gene sets specific to ascites inflammatory DCs and inflammatory macrophages. Given that $C D 1 C$ mRNA was elevated in CRIg ${ }^{\text {lo }}$ cells by RNA sequencing, we used GSEA to compare ascites fluid macrophages to the profiles identified by Segura et al. The macrophage signature was strongly enriched in CRIghi macrophages (NES 2.81, FDR q value 0.0), while the inflammatory DC signature was enriched in $\mathrm{CRIg}^{\mathrm{lo}}$ macrophages (CD1C+: NES 1.98, FDR q value 0.0) (Supplemental Figure 2, A and B).

We used the Functional Annotation Clustering tool in DAVID (30) to identify significantly enriched pathways and gene ontologies in CRIghi or CRIg ${ }^{\text {lo }}$ macrophages. Lysosomal and cell cycle pathways were strongly enriched in CRIghi macrophages, whereas the CRIg ${ }^{\text {lo }}$ macrophage expression signature was related to the inflammatory response (Figure 5E). Lysosome was also the most highly enriched ontology in murine $\mathrm{F} 4 / 80^{\text {hi }}$ resident macrophages compared with inflammatory macrophages (13).

While few studies have examined human tissue macrophage ontogeny, human macrophage activation states are relatively well studied. Xue et al. recently characterized the human macrophage activation spectrum by in vitro stimulation of monocyte-derived macrophages with a diverse range of inflammatory stimuli (31). We used GSEA to investigate whether any of the 49 activation modules identified by Xue et al. were enriched in ascites fluid macrophages. Ten modules were significantly enriched in CRIg ${ }^{\mathrm{h}}$ macrophages, which were associated with glucocorticoid-, IL-4/IL-13-, and lipid-activated macrophage signatures. Three modules associated with lipid-, IL-4/IL-13-, and TNF and PGE2-activated macrophage signatures were significantly enriched in CRIg ${ }^{\text {lo }}$ macrophages (Supplemental Table 3). CRIghi and CRIglo enriched genes ( $\geq 3$-fold) formed distinct clusters within the human macrophage activation spectrum, illustrating that in vivo macrophages exhibit a complex mixture of activation signatures and further highlighting the association among CRIg ${ }^{10}$-enriched genes and inflammatory stimuli and CRIghi-enriched genes with antiinflammatory stimuli (Supplemental Figure 2C).

Relationship between ascites fluid macrophages and mouse peritoneal macrophages. We used GSEA to compare 
A

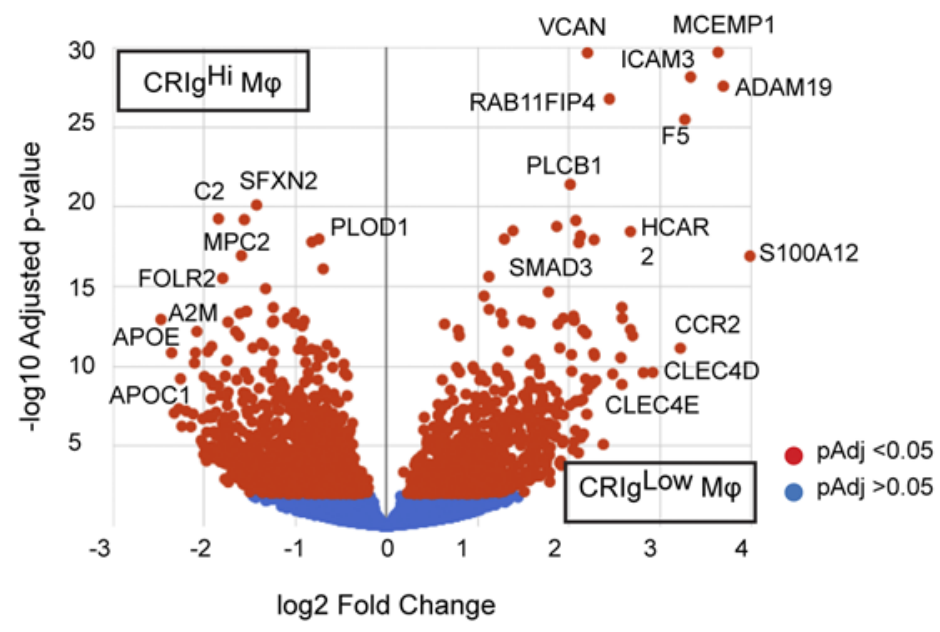

C Genelist: Higher in 7 day cultured monocytes

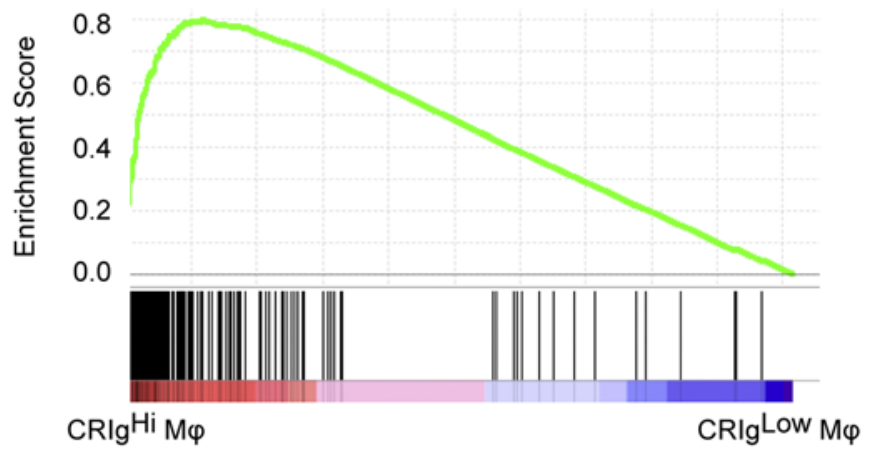

B

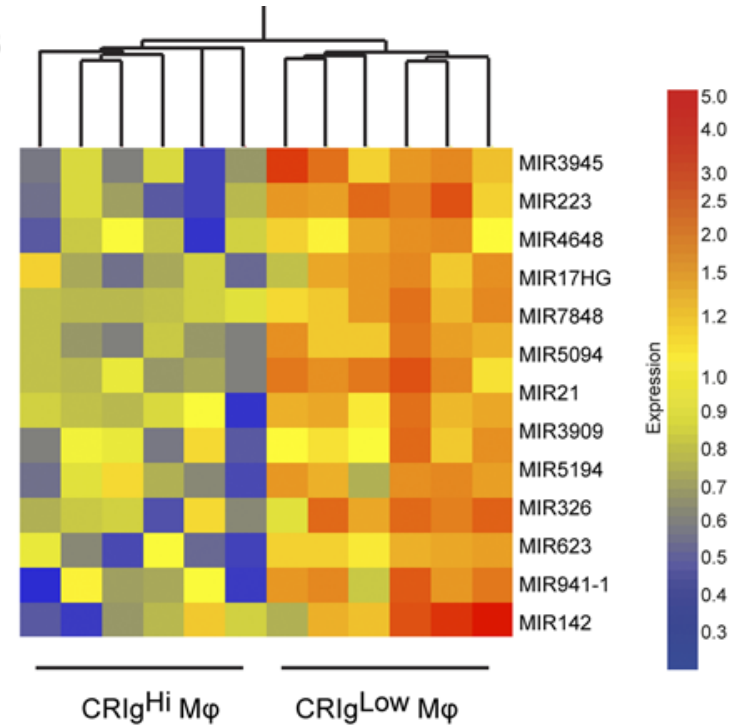

$\mathbf{E}$

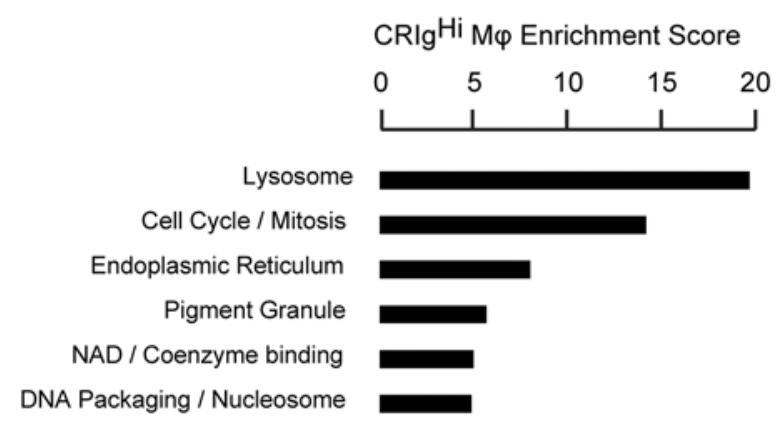

D Genelist: Higher in uncultured monocytes
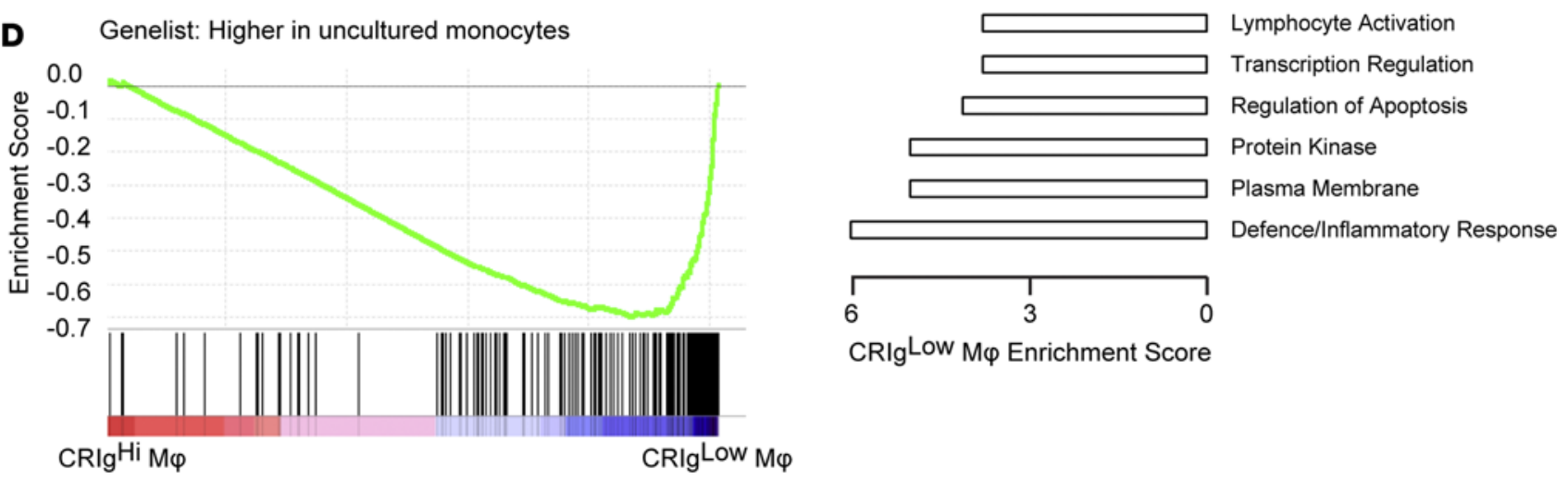

Figure 5. Transcriptional profiling of ascites macrophages by RNA sequencing. (A) Volcano plot illustrating genes significantly increased in CRIghi or CRIg ${ }^{\text {lo }}$ ascites macrophages by RNA sequencing ( $n=6$ patients, DESeq2-generated adjusted $P$ value and fold change). (B) Differentially expressed microRNAs (MIR) and macrophage samples were clustered by distance correlation. (C) Macrophage and (D) monocyte gene set enrichment with respect to ascites fluid macrophage profile. Ascites macrophage data are ranked according to differential expression between subpopulations (indicated by red-blue bars), and the gene set of interest is mapped onto this profile (black bars) to determine enrichment score (green lines). (E) Functional clusters enriched in CRIghi or $\mathrm{CRIg}{ }^{10}$ macrophages by representative ontology term. The enrichment score represents the log(geometric mean) of the individual enrichment $P$ values of members within a functional cluster. A higher enrichment score indicates consistently lower $P$ values for the clustered terms.

ascites fluid macrophage profiles to murine peritoneal macrophage signatures available through the Immunological Genome Project $(12,21)$. Genes were classified as subset enriched if they were $\geq 2$-fold more highly expressed (Benjamini-Hochberg-corrected $P<0.05$ ) in one population (i.e., $\mathrm{F} 4 / 80^{\text {hi }}$ with or without thioglycollate, $\mathrm{F} 4 / 80^{\text {lo }}$ with or without thioglycollate, $\mathrm{F} 4 / 80^{\text {Int. }}$ with or without MHCII expression) com- 
A Geneset: F4/80 $\mathrm{Hi}$. (Basal OR Thio.)

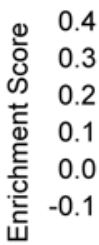
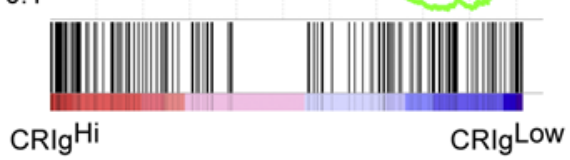

C Geneset: Upregulated in Gata6 KO M $\varphi$

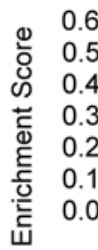

|||||||||||||||||| | ||||||||||||||||||||||

CRIgHi

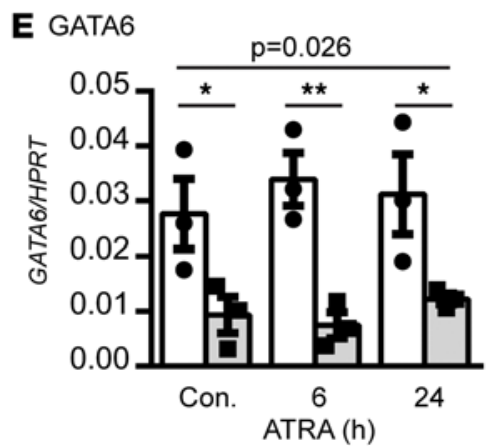

H LRG1

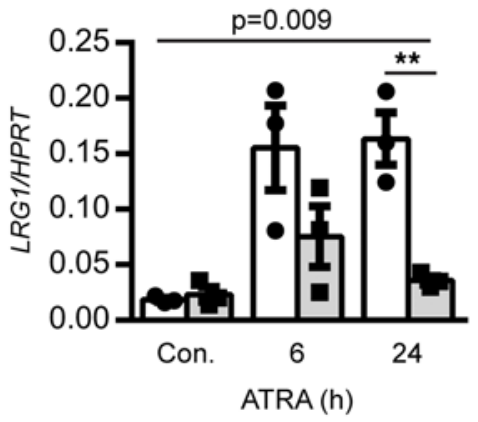

F TGFB2

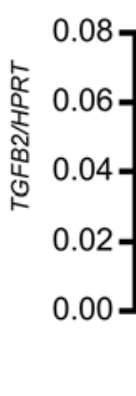

I RARB

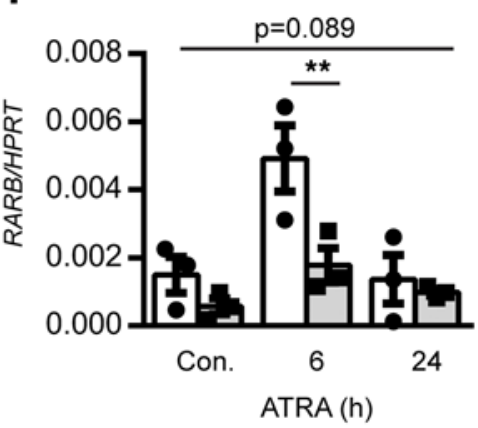

L CD49F (ITGA6)

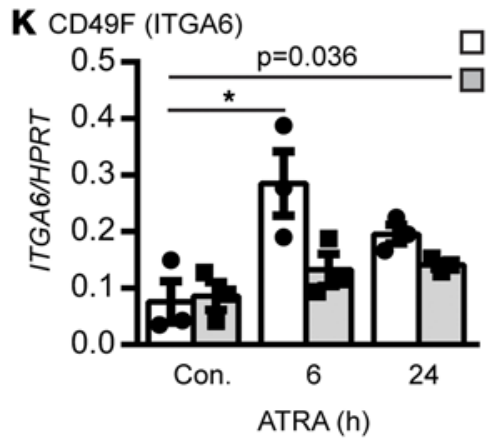

CRIgLow M $\varphi$

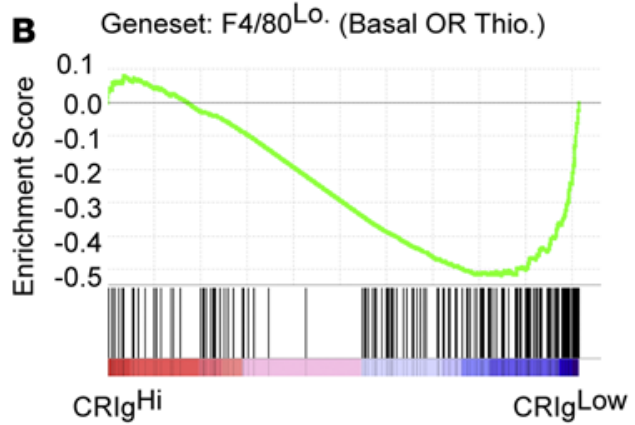

D Geneset: Downregulated in Gata6 $\mathrm{KO} M \varphi$

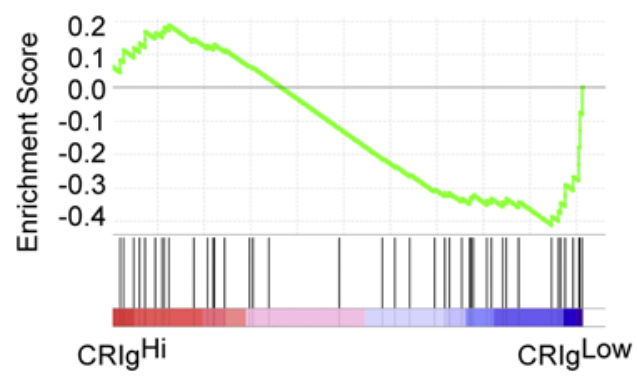

\section{G $\mathrm{CXCL} 13$}
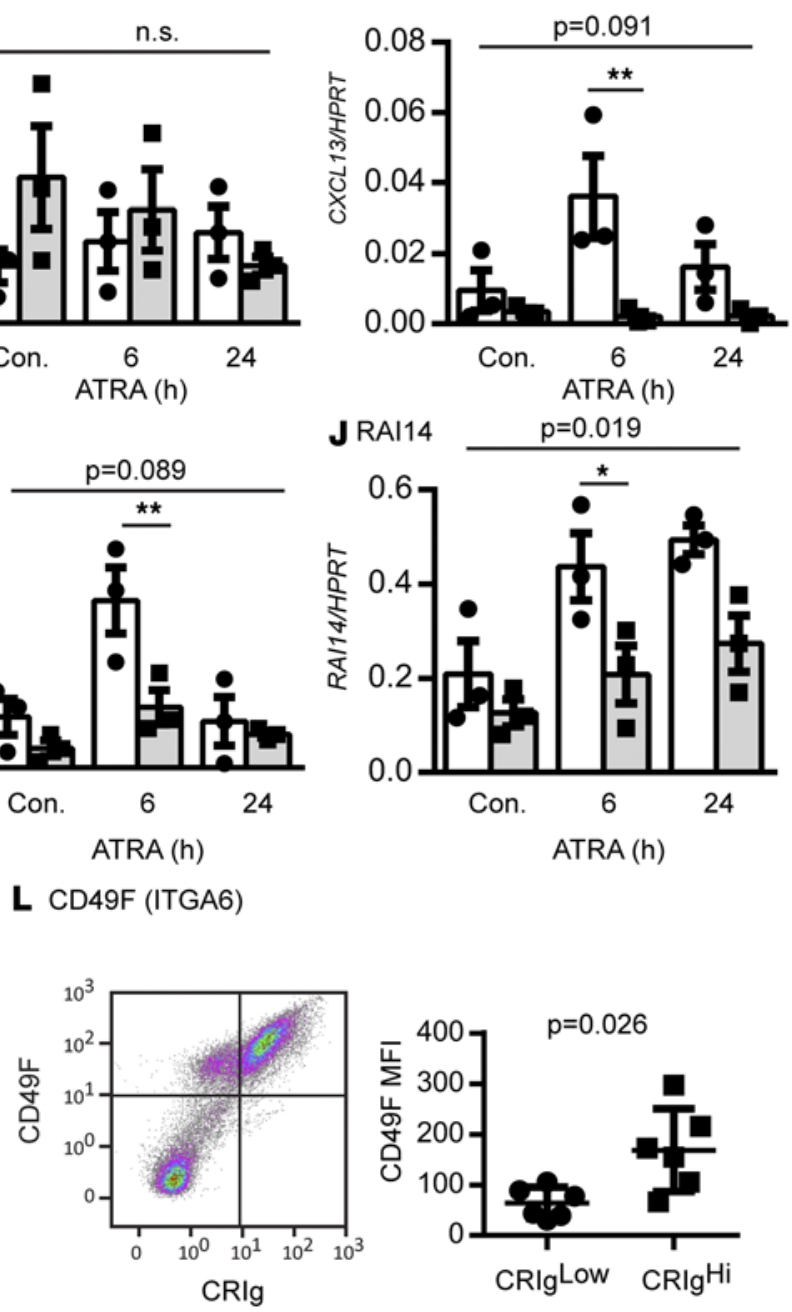
Figure 6. Relationship between human and murine peritoneal macrophage signatures. (A and B) Murine peritoneal macrophage-specific gene set enrichment with respect to the ascites fluid macrophage profile. Ascites macrophage data are ranked according to differential expression between subpopulations (indicated by red-blue bars), and the gene set of interest is mapped onto this profile (black bars) to determine enrichment score (green lines) (Thio., thioglycollate). Enrichment of genes (C) upregulated and (D) downregulated in murine Gata6-deficient macrophages in ascites macrophages (GSEA analysis as in A). Relative expression of (E) GATA6, (F) TCFB2, (G) CXCL13, (H) LRG1, (I) RARB, (J) RAl14, and (K) CD49F in CRIg ${ }^{\text {hi }}$ and CRIg ${ }^{10}$ macrophages stimulated with ATRA ( $n=3$, repeated-measures 2-way ANOVA followed by Sidak multiple comparison test for differences between macrophage populations or time point). (A-J) ANOVA $P$ values for the effect of macrophage population or (K) ATRA stimulation on gene expression; ${ }^{*} P<0.05,{ }^{* *} P<0.01$. (L) Mean fluorescence intensity (MFI) of CD49F surface expression on ascites macrophage populations (Mann-Whitney test, $n=6$ ) and representative dot plot. Dot plot data represent mean \pm SEM.

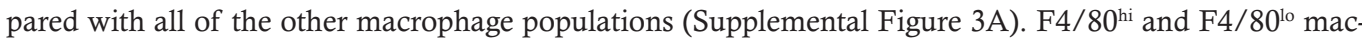
rophages were defined by the expression of 189 and 227 genes, respectively; however, only 21 genes were uniquely enriched in F4/80 Int. macrophages, precluding GSEA of this population. The F4/80 $0^{\text {hi }}$ expression signatures (with or without thioglycollate) were significantly enriched in CRIghi macrophages (NES 1.62, FDR q value 0.013 ). By contrast, the F4/80 10 expression signatures (with or without thioglycollate) were enriched in CRIglo macrophages (NES 1.99, FDR q value 0.0) (Figure 6, A and B, and Supplemental Table 4). Given the most prominent activation signature in both populations of ascites macrophages related to IL-4 stimulation, we further analyzed the relationship between ascites macrophages and alternatively activated resident (induced by IL-4 stimulation) and monocyte-derived (induced by thioglycollate and IL-4 stimulation) murine peritoneal macrophages $(32,33)$, compared with unstimulated and thioglycollate-stimulated macrophages. Genes were classified as enriched if they were $\geq 2$-fold more highly expressed (Benjamini-Hochberg-corrected $P<0.05$ ) in one treatment compared with all of the other 3 treatments (Supplemental Figure 3B). Consistent with the similarity between CRIghi ascites macrophages and murine resident $\left(\mathrm{F} 4 / 80^{\text {hi }}\right)$ macrophages and the alternative activation signature, the alternatively activated resident macrophage signature was highly enriched in CRIghi macrophages (NES 2.6, FDR q value 0.0), while the alternatively activated monocyte-derived macrophage signature was modestly enriched in CRIglo macrophages (NES 1.3, FDR q value 0.09; Supplemental Figure 3D).

Gata6, a transcription factor that selectively regulates $\mathrm{F} 4 / 80^{\mathrm{hi}}$ resident peritoneal macrophage expression and function in mice (14), was expressed at relatively low levels by RNA sequencing in both ascites macrophage populations but was approximately 2-fold more highly expressed in CRIg ${ }^{\text {lo }}$ macrophages (adjusted $P=0.08$, Supplemental Table 1). At a global level, genes that were upregulated in murine Gata6 knockout macrophages, which were associated with alternative activation (11), were strongly enriched in CRIgh macrophages (NES 2.23, FDR q value 0.0, Figure 6C). By contrast, genes that were downregulated in Gata6 knockout mice, which include peritoneal macrophage-specific transcripts $(11,13)$, were modestly enriched in CRIgio macrophages (NES 1.28, FDR q value 0.088, Figure 6D). Interestingly, E2F and MYC target genes were highly enriched in CRIghi macrophages (NES 2.21, FDR q value 0.0 and NES 1.77, FDR $\mathrm{q}$ value 0.0 , respectively), suggesting a role for these transcription factors in CRIghi macrophage development or activation.

Gata6 expression in mouse $\mathrm{F} 4 / 80^{\text {hi }}$ resident peritoneal macrophages, and their capacity to regulate gut IgA production through peritoneal B-1 cells, was recently shown to be dependent on vitamin A signaling (14). The vitamin A axis is of significant interest and potential clinical relevance in the setting of cirrhosis, as these patients have impaired mucosal immunity that likely contributes to peritoneal bacterial translocation (34) and are frequently vitamin A deficient (35); however, vitamin A signaling in human peritoneal macrophages has not been investigated. We treated flow cytometry-sorted CRIghi and CRIg ${ }^{\text {lo }}$ macrophages with all-trans retinoic acid (ATRA) for 6 or 24 hours and quantified expression of murine retinoic acid responsive genes (14). GATA6 mRNA was more highly expressed in cultured CRIg ${ }^{\text {lo }}$ macrophages compared with CRIghi macrophages but was not induced by ATRA (Figure 6E). Similarly, in mice, Gata6 is only retinoic acid inducible in fetal, but not adult, macrophages, and it is postulated that mature macrophages have undergone epigenetic changes that prevent Gata6 induction $(14,36)$. With the exception of TGFB2 and $A R G 1$ (which was not detected by RNA sequencing or qPCR), all selected genes (CXCL13, LGR1, RARB, $R A I 14$, and ITGA6 [CD49F]) were more highly expressed and/or ATRA inducible in CRIglo macrophages compared with CRIghi macrophages (Figure 6, F-K). Although not differentially expressed in unstimulated, sorted macrophage populations at the transcript level (Figure 6K), surface CD49F (a Gata6 target in mice; ref. 14) was elevated on CRIghi cells (Figure 6L). Together, these data suggest a more prominent role for retinoic acid signaling in CRIg ${ }^{\text {lo }}$ macrophages compared with CRIghi macrophages, although the role of GATA6 
is not known. In support of this, we performed a connectivity map analysis (37) to identify potential drugs that induced the CRIghi or CRIg ${ }^{\text {lo }}$ macrophage signature, and the strongest drug class association was the topical retinoids (D10AD), predicted to favor the CRIglo macrophage profile $(P=0.00046)$.

\section{Discussion}

New strategies are needed to prevent recurrent infections, minimize the use of empirical antibiotics, and improve outcomes for patients with cirrhosis. Therapeutic manipulation of the host immune response may be a viable strategy; however, the mechanisms and clinical relevance of peritoneal immune function and dysfunction require clarification. Although acute and chronic peritoneal inflammation has been extensively studied in mice, and developmentally distinct resident and inflammatory peritoneal macrophages have been characterized in detail, human peritoneal macrophages have not been widely studied. Here, we have identified 2 phenotypically and functionally distinct human peritoneal macrophage subpopulations in ascites fluid from cirrhotic patients, in the absence of overt infection, which can be clearly distinguished on the basis of CRIg and CCR2 expression. Our data suggest the presence of CRIghi macrophages (or absence of CRIg ${ }^{\text {lo }}$ macrophages) is associated with improved liver status and better prognosis. Future studies will be required to determine the peritoneal macrophage phenotype in noncirrhotic patients, in order to understand whether $\mathrm{CRIg}^{\text {hi }} \mathrm{CCR} 2^{-}$and $\mathrm{CRIg}{ }^{\mathrm{lo}} \mathrm{CCR} 2^{+}$cells are developmentally distinct and/or shaped by peritoneal inflammation.

If monocyte-dependent and -independent macrophages are present in ascites fluid as in the mouse peritoneum, in health and disease, several lines of evidence support the hypothesis that CRIghi macrophages are, or contain, the human counterparts of murine F4/80 hi resident peritoneal macrophages. First, F4/80 hi macrophages express high levels of CRIg/Vsig4; whereas Ccr2, that defines CRIglo macrophages, is expressed on monocyte-derived macrophages $(13,14)$. Second, at a transcriptomic level, when compared with human immune cell expression profiles, CRIghi macrophages were highly enriched for mature macrophage transcripts and resembled macrophages from human tumor ascites fluid, whereas CRIg ${ }^{\text {lo }}$ cells exhibited a monocytic signature and resembled inflammatory monocyte-derived DCs found in tumor ascites fluid (29). Similarly, CRIghi macrophages were significantly, albeit modestly, enriched for genes that define $\mathrm{F} 4 / 80^{\mathrm{hi}}$ mouse macrophages, while the $\mathrm{F} 4 / 80^{\mathrm{lo}}$ macrophage expression signature was enriched in the CRIglo population. Third, the strong cell cycle signature in CRIghi macrophages is consistent with the hypothesis that these cells are a self-renewing, tissue-resident population (15) - although it should be noted that both bone marrow-derived and tissue-resident macrophage lineages proliferate at different stages during inflammation in mice $(38,39)$.

Despite the similarities between CRIghi and F4/80 hi macrophages, some of our data point to similarities between $\mathrm{CRIg}^{\mathrm{lo}}$ and $\mathrm{F} 4 / 80^{\mathrm{hi}}$ macrophages. GATA6, a master transcriptional regulator of $\mathrm{F} 4 / 80^{\mathrm{hi}}$ resident macrophages $(11,13,14)$, was more highly expressed in CRIglo macrophages compared with $\mathrm{CRIg}^{\text {hi }}$ ascites macrophages, as were murine Gata6-dependent genes (11). Expression of Gata6 itself and several of its target genes is vitamin A-dependent in mouse macrophages (14). Four of six selected murine Gata6/retinoic acid target genes were also ATRA inducible in ascites macrophages, all of which were more highly expressed/inducible in CRIg ${ }^{\text {lo }}$ macrophages compared with CRIg ${ }^{\text {hi }}$ macrophages. If GATA6 fulfils the same function in humans as in mice, these data suggest an alternative hypothesis - that CRIghi and CRIglo macrophages are subsets of one major human resident peritoneal population that is equivalent to murine $\mathrm{F} 4 / 80^{\mathrm{hi}}$ macrophages, since both populations express VSIG4 and GATA6, although at different levels. CRIghi macrophages may upregulate CRIg, and downregulate CCR2 and GATA6, during maturation in the ascites microenvironment. Indeed, we observed surface CRIg upregulation when ascites cells were incubated with E. coli bioparticles, especially in autologous ascites fluid. Similarly in mice, zymosan-induced peritonitis upregulated Vsig4 mRNA (13), and thioglycollate activation also induced Vsig4 in F4/80 resident peritoneal macrophages (GSE15907; ref. 21). Finally, if GATA6 does not define monocyte-independent peritoneal macrophages in humans, it is possible that the ascites macrophages studied here are monocyte derived, akin to the $\mathrm{F} 4 / 80^{\mathrm{lo}}$ murine population, and the omentum-derived resident population, if it exists, remains to be identified. Future investigations, potentially using additional markers to those identified here, will be important to refine ascites macrophage phenotypes and clarify their origin and function to confirm their relationship to murine peritoneal macrophages.

Our data suggest that ascites fluid macrophages are associated with clinical parameters and potentially clinical outcomes. An increased proportion of CRIghi macrophages in ascites fluid, and a reciprocally 
low proportion of CRIglo macrophages, correlated with lower MELD scores. In mice, F4/80 ${ }^{\text {hi }}$ resident macrophages disappear during acute inflammation, while infiltrating monocytes augment the macrophage compartment, so alterations in relative macrophage proportions may simply be indicative of peritoneal inflammation in patients. CRIg itself is a multifunctional protein that promotes phagocytosis of C3-opsonized microbes and apoptotic cells in conjunction with CR3, enhances killing of intracellular bacteria (40), and also possesses antiinflammatory and immunosuppressive functions $(19,20,22,41-44)$. CRIghi macrophages may beneficial due to their superior phagocytic and microbicidal capacity, due to other antiinflammatory functions mediated by CRIg, or for reasons unrelated to CRIg expression. CRIghi macrophages were enriched for IL-4-related human macrophage activation modules (31), the murine alternatively activated resident macrophage signature (33), and genes that were upregulated in murine Gata6 knockout macrophages, which have been associated with alternative activation (11). Given their high phagocytic capacity and antiinflammatory activation signature, the data could suggest that CRIghi macrophages are "alternatively activated," which may relate to the negative correlation between CRIghi macrophages and MELD score. Alternative activation, a critical component of type 2 immunity, also contributes to the maintenance of tissue and metabolic homeostasis (45). Additionally, or alternatively, CRIglo macrophages may be disadvantageous due to their inflammatory nature and reduced phagocytic and microbicidal function. In addition to the implications for host defence, sustained peritoneal inflammation may also contribute to impaired lymphatic function and peritoneal fluid drainage, which was promoted by infiltrating, inflammatory Ccr2-dependent macrophages in a mouse model of peritonitis $(46,47)$.

Consistent with CRIg's antiinflammatory functions, VSIG4 gene expression is upregulated by antiinflammatory stimuli such as IL-10 and dexamethasone and downregulated by proinflammatory stimuli such as IFN- $\gamma$ and TNF in vitro and in vivo (48-50). Although increased transcription and translation could conceivably contribute to the CRIg induction we observed in ascites fluid, other as-yet-unknown factors regulating surface CRIg expression are likely involved. Importantly, these data suggest the importance of modifiable factors that could potentially be harnessed to enhance peritoneal macrophage function in cirrhotic patients. CSF-1 controls proliferation of resident and recruited peritoneal macrophages in mice, and IL-4 may also play a role in some settings $(38,51)$. Ascites macrophages expressed both CSF1R and IL4R, with higher levels of CSF1R mRNA and surface expression (data not shown) in CRIghi macrophages. Given that CSF-1 has been extensively developed as a therapeutic target (52) and was recently associated with good outcomes in patients with acute liver failure and demonstrated to promote liver regeneration in mice (53), CSF-1 treatment may represent a viable therapeutic strategy. Interestingly, CRIghi macrophages exhibited a prominent IL-4 activation signature, although this was observed in both ascites macrophage populations. In contrast to the role of vitamin A in promoting intestinal homeostasis in mice, our data suggest that vitamin A signaling favors the CRIg ${ }^{\text {lo }}$ macrophage profile, which was associated with higher MELD scores in our cohort. However, understanding the contribution of macrophage origin to eventual function, and the relative contributions of recruitment and proliferation to inflammatory responses, will be critical for macrophage-targeted therapy.

In conclusion, we report the presence of distinct peritoneal macrophage subpopulations, which may contribute to the poor outcomes experienced by patients with cirrhosis and ascites. Understanding the dynamics, functions, and clinical relevance of these populations may provide novel therapeutic approaches to improve clinical outcomes.

\section{Methods}

Patients and ascites fluid collection. Ascitic fluid (2-4 liters) was collected from patients with decompensated cirrhosis undergoing therapeutic paracentesis at the Princess Alexandra Hospital. Standard biochemical and serological assays, liver imaging, and histological assessment of a liver biopsy (if performed) were used to confirm diagnosis of liver disease and cirrhosis. Clinical data were obtained by review of patient medical records. Liver disease severity was evaluated using $\operatorname{MELD}(3.78 \times \ln [$ serum bilirubin $\{\mathrm{mg} / \mathrm{dL}\}]+11.2 \times \ln [\mathrm{INR}]+9.57 \times \ln [\mathrm{se}-$ rum creatinine $\{\mathrm{mg} / \mathrm{dL}\}]+6.43$; ref. 54). Of the 6 patients selected for RNA sequencing, all were of mixed European descent, mean age was 62.9 (SD 8.9), 4 were male, 3 had alcoholic liver disease, 2 had nonalcoholic steatohepatitis, and 1 had chronic HCV infection complicated by alcohol use. Ascites fluid cells were harvested by centrifugation (400 $\mathrm{g}$ for 5 minutes) and cryopreserved in medium containing $90 \% \mathrm{FBS} / 10 \%$ DMSO.

Flow cytometry. For cell phenotyping, $0.5 \times 10^{6}$ to $1 \times 10^{6}$ cells were resuspended in FACS buffer (PBS/1\% FBS/5 mM EDTA) and stained with optimized panels comprising combinations of the following antibodies, with the addition of Live/Dead Aqua (Thermo Fisher) for dead cell discrimination in some 
experiments, on ice for 30 minutes in the dark: CD14-BV421 (M5E2), CD14-APC (M5E2), CD163-PerCPCy5.5 (GHI/61), HLA-DR-FITC (L243), CD62L-FITC (DREG-56), SIGLEC-1-APC (7-239), CD11BAPC (ICRF44), and CSF1R-PE (9-4D2-1E4) (all BioLegend); CD16-APC-H7 (3G8; BD Biosciences); CCR2-APC (48607) and CD49F-AF488 (GoH3) (both R\&D Systems); and CRIg-PE (6H8, Santa Cruz). Samples were washed in FACS buffer and resuspended in FACS buffer containing 1\% PFA for analysis. Isotype controls were employed during panel development (Supplemental Figure 1), and unstained and single color controls were routinely performed to generate compensation matrices to correct for spectral overlap and facilitate gating. Samples were acquired on a Gallios flow cytometer, and data were analyzed using Kaluza software (Beckman Coulter). For cell sorting, cells were labeled with CD14-BV421 or CD14APC and CRIg-PE and sorted into FBS-coated polystyrene tubes using a MoFlo Astrios sorter (Beckman Coulter), achieving $>90 \%$ purity. Since CRIg staining did not completely separate the populations in every patient sample, we routinely sorted CRIghi and CRIg ${ }^{\text {lo }}$ cells, avoiding the region of potential overlap between the populations. For analysis and sorting, dead cells/debris were excluded by their low FSC/high SSC profile or high Live/Dead Aqua (Life Technologies) staining, and doublets were excluded using FSCHeight versus FSC-Area or time-of-flight. Negative gates were set based on unstained samples and compensated single color controls (Supplemental Figure 1). CD14 was used to define monocytes/macrophages for quantitation of surface marker expression, although plots show all ascites leukocytes for reference.

Opsonization, phagocytosis, and infection assays. pHrodo E. coli BioParticles (Molecular Probes) were added to ascites cells in 5-ml flow cytometry tubes at a ratio of $10: 1$ and incubated at $37^{\circ} \mathrm{C}$ for 1 hour, with antibodies to cell surface markers added after 30 minutes. Cells were washed twice with cold FACS buffer prior to acquisition. FitC-labeled carboxylated polystyrene beads ( $2 \mu \mathrm{m}$; Sigma-Aldrich) were diluted to $0.5 \%$ solids in PBS, human IgG (10 mg/ml; Sigma-Aldrich), human AB serum (Sigma-Aldrich), or HI AB serum $\left(45\right.$ minutes at $\left.56^{\circ} \mathrm{C}\right)$ and incubated for 2 hours at $37^{\circ} \mathrm{C}$. Beads were added to ascites cells resuspended in serum-free DMEM, pretreated for 20 minutes with $10 \mu \mathrm{g} / \mathrm{ml}$ Cytochalasin D (Sigma-Aldrich) or left untreated, at a ratio of $10: 1$ and incubated for 1 hour at $37^{\circ} \mathrm{C}$, with the addition of antibodies to cell surface markers after 30 minutes. Cells were washed twice with cold FACS buffer and resuspended in FACS buffer with 1\% PFA for analysis. Bead and bioparticle phagocytosis were analyzed using a Gallios flow cytometer and Kaluza analysis software (Beckman Coulter). Complement C3 deposition following serum opsonization was confirmed by staining opsonized beads with anti-human C3 (1H8, Cedarlane) followed by an antimouse Alexa647 secondary antibody (Molecular Probes) and analyzed by flow cytometry.

Bacterial infection assays were conducted in HMDMs and CRIghi/lo macrophages as previously described (55). In brief, in vitro-differentiated HMDMs or flow cytometry-sorted ascites cells were cultured at $2 \times 10^{5}$ cells/well in $1 \mathrm{~mL}$ antibiotic-free tissue culture media at $37^{\circ} \mathrm{C}$ overnight prior to infection with $E$. coli (strain MG1655) at a MOI of 10 . At 1 hour after infection, cells were washed with media containing $200 \mu \mathrm{g} / \mathrm{mL}$ gentamicin (gentamedia) to exclude extracellular bacteria and then maintained for 1 hour in $200 \mu \mathrm{g} / \mathrm{mL}$ gentamedia. Cells were then washed with and maintained in $20 \mu \mathrm{g} / \mathrm{mL}$ gentamedia for a further 3 hours. Cells were harvested in $0.01 \%$ Triton X-100 in PBS and lysed by pipetting. Diluted lysates were cultured on $\mathrm{LB}$ agar plates at $37^{\circ} \mathrm{C}$ overnight, and colony counts were used to assess intracellular bacterial loads. Culture supernatants were collected to assess cell death through the release of the metabolic enzyme, LDH, from dying cells into culture supernatants. LDH release was quantified by measuring LDH in culture supernatants in comparison to total LDH (LDH present in supernatants from untreated cells following cell lysis with $0.1 \%$ Triton X-100), as previously described (56).

RNA purification, ATRA stimulation, and $q P C R$. Total RNA was purified using RNA Nucleospin columns (Machery Nagel). For ATRA stimulation, flow cytometry-sorted CRIghi and CRIglo macrophages were seeded in 12 -well plates $\left(0.5 \mathrm{e}^{6} /\right.$ well) in RPMI containing $10 \% \mathrm{FBS}$ and Penicillin/Streptomycin and allowed to adhere for 2 to 4 hours. Cells were treated with $10 \mu \mathrm{M}$ ATRA (Sigma-Aldrich) or vehicle control (DMSO, Sigma-Aldrich) 24 or 6 hours prior to harvest in lysis buffer for RNA purification. cDNA was synthesized using Superscript III (Invitrogen) with oligo dT priming. qPCR was performed using SYBR Green PCR Mastermix (Applied Biosystems) and a ViiA 7 real-time PCR system (Applied Biosystems). Gene expression relative to hypoxanthine-guaninephosphoribosyltransferase (HPRT) was quantified using the $\Delta \mathrm{Ct}$ method. The following primers were used in this study: HPRT-F TGCTGGATTACATCAAAGCACTG, HPRT-R CCCCTGTTGACTGGTCATTACAA, LRG1-F CAGGACAACCCAGAAGCAAAAG, LRG1-R TCTAGAAACATGGGGTTGAATGC, ARG1-F TCAACACTCCACTGACAACCACA, ARG1-R AATCCTGGCACATCGGGAATC, GATA6-F ACTTCCCCCACAACACAACCT, 
GATA6-R AGCTCGCTGTTCTCGGGATT, RAI14-F CAGCAAGTCAAACAGCTCCAGA, RAI14-R GCCCTGCACAGCATACAGAAG， RARB-F ATCAGAAAAAGACGACCCAGCA， RARB-R AGGTAATTACACGCTCTGCACCTT， CD49F-F TATTCGGGAGTACCTTGGTGGA， CD49F-R AGCGTTTAAAGAATCCACACTTCC， TGFB2-F GGATGCGGCCTATTGCTTTAGA， TGFB2-R TCGTGTATCCATTTCCACCCTAGA，CXCL13-F AATTGTGTGTGTGGACCCTCAA，CXCL13-R GGGAATCTTTCTCTTAAACACTGGAA.

RNA sequencing and data analysis. RNA sequencing was performed by the University of Queensland IMB Sequencing Facility. Libraries were generated from 500 ng total RNA (Agilent Bioanalyzer RNA Integrity values $\geq 8$ ) using TruSeq Ribo-Zero GOLD RNA Library Preparation Kit Set A and Set B (Illumina, catalog RS-122-2301 and RS-122-2302) and sequenced on a $1 \times 75$ bp High Output V2 run (Illumina, catalog FC-404-2005) using a NextSeq500 sequencing system (Illumina, NextSeq control software v1.4/ Real Time Analysis v2.1). A total of 475.7 million reads were obtained, with 28.3 million to 58.2 million reads per replicate, and $95.3 \%$ of base calls achieved an Illumina $\mathrm{Q}$ score $\geq \mathrm{Q} 30$. Sequencing reads were mapped to UCSC human genome version hg19, using STAR v2.3.0e (57) with 25.7 million to 52.3 million uniquely mapped reads per replicate, and the SAM output was converted to BAM format using samtools v0.1.19 (58). Gene read counts were obtained using the HTSeq python package (59). Differential expression was detected using the DESeq2 (60) and EdgeR (24) R packages and filtered to remove genes with $<2$ counts in any replicate (retaining 13,431 expressed genes). Differentially expressed genes $(n=2,348)$ were defined as genes that were identified as differentially expressed using both the DESeq2 (adjusted $P<$ $0.05, n=3,634)$ and EdgeR (FDR $<0.05, n=2,373$ ) algorithms. The RNA sequencing data are available in the GEO repository under accession number GSE79833. GSEA was performed using GSEA v 2.10 using gene set permutation and default settings. Gene sets used for analysis were from immune collection from the MSIG database (27), Segura et al. (29), Xue et al. (31), Gautier et al. (11), Gundra et al. (33), and the Immgen consortium (21) (see below). Gene ontology analysis was performed using DAVID (https:// david.ncifcrf.gov/) with default parameters. Connectivity map analysis was performed using the tool at http://www.broadinstitute.org/cmap (37) with default parameters. To identify murine peritoneal macrophage subset-enriched genes, samples corresponding to the major ( F4/80 $\left.0^{\text {hi }} \mathrm{MHCII}\right)$ and minor $\left(\mathrm{F} 4 / 80^{\mathrm{lo}} \mathrm{M}\right.$ $\mathrm{HCII}^{+}$) resident populations under basal conditions and following thioglycollate activation as well as the infiltrating $\mathrm{F} 4 / 80^{\text {Int }} \cdot \mathrm{MHCII}^{+}$and $\mathrm{F} 4 / 80^{\text {Int. }} \mathrm{MHCII}^{-}$populations were downloaded from the Immunological Genome Project (GSE15907; ref. 21) and analyzed using Genespring GX. Data from GSE54679 (33) were analyzed to identify genes enriched in unstimulated, thioglycollate-activated, IL-4-activated, and IL-4 and thioglycollate-activated murine peritoneal macrophages using Genespring GX.

Statistics. Statistical analyses were performed in Graph Pad Prism (version 6.04; GraphPad Software Inc.), utilizing the Mann-Whitney test for pairwise comparisons, ordinary and repeated-measures 2-way ANOVA with Tukey's multiple comparisons test for multiple comparisons, 1-sample $t$ test for data normalized to control (comparison to the hypothetical mean of 1.0), and Spearman's rank-correlation coefficient. All comparisons were 2-tailed and a $P$ value of less than 0.05 was defined as the level of significance. All data are represented as mean \pm SEM.

Study approval. Informed written consent was obtained from each patient prior to inclusion in the study, and the protocol was approved by the Metro South Health and The University of Queensland Human Research Ethics Committees.

\section{Author contributions}

$\mathrm{KMI}$ conceived and designed the study, collected and analyzed data, and wrote the manuscript. XB, KKW, VLG, JKA, and SJ collected samples and data. SL and GJB analyzed data. MJS designed experiments and critically reviewed the manuscript. EEP supervised the study and wrote the manuscript.

\section{Acknowledgments}

We wish to thank the staff at Princess Alexandra Hospital, the patients who participated, and the Translational Research Institute flow cytometry core facility. This study was funded by the PA Hospital Foundation, an Australian Liver Foundation Pauline Hall Fellowship to K.M. Irvine, a National Health and Medical Research Foundation Practitioner Fellowship to E.E. Powell (APP1004242), and a National Health and Medical Research Foundation Senior Research Fellowship (APP1107914) to M.J. Sweet. 
Address correspondence to: Katharine Irvine, Centre for Liver Disease Research, School of Medicine, The University of Queensland, Translational Research Institute, 37 Kent St., Brisbane, 4102, Australia. Phone: 61.7.3443.8014; E-mail: katharine.irvine@gmail.com.

1. Fagan KJ, et al. The burden of decompensated cirrhosis and ascites on hospital services in a tertiary care facility: time for change? Intern Med J. 2014;44(9):865-872.

2. Arvaniti V, et al. Infections in patients with cirrhosis increase mortality four-fold and should be used in determining prognosis Gastroenterology. 2010;139(4):1246-1256.

3. Lim KH, Potts JR, Chetwood J, Goubet S, Verma S. Long-term outcomes after hospitalization with spontaneous bacterial peritonitis. J Dig Dis. 2015;16(4):228-240.

4. Wiest R, Lawson M, Geuking M. Pathological bacterial translocation in liver cirrhosis. J Hepatol. 2014;60(1):197-209.

5. Cholongitas E, Papatheodoridis GV, Lahanas A, Xanthaki A, Kontou-Kastellanou C, Archimandritis AJ. Increasing frequency of Gram-positive bacteria in spontaneous bacterial peritonitis. Liver Int. 2005;25(1):57-61.

6. Quigley EM, Stanton C, Murphy EF. The gut microbiota and the liver. Pathophysiological and clinical implications. J Hepatol. 2013;58(5):1020-1027.

7. Fagan KJ, et al. Ascites bacterial burden and immune cell profile are associated with poor clinical outcomes in the absence of overt infection. PLoS One. 2015;10(3):e0120642.

8. Leentjens J, et al. Reversal of immunoparalysis in humans in vivo: a double-blind, placebo-controlled, randomized pilot study. Am J Respir Crit Care Med. 2012;186(9):838-845

9. Garcia-Gonzalez M, Boixeda D, Herrero D, Burgaleta C. Effect of granulocyte-macrophage colony-stimulating factor on leukocyte function in cirrhosis. Gastroenterology. 1993;105(2):527-531

10. Ghosn EE, et al. Two physically, functionally, and developmentally distinct peritoneal macrophage subsets. Proc Natl Acad Sci US A. 2010;107(6):2568-2573.

11. Gautier EL, et al. Gata6 regulates aspartoacylase expression in resident peritoneal macrophages and controls their survival. $J$ Exp Med. 2014;211(8):1525-1531.

12. Gautier EL, et al. Gene-expression profiles and transcriptional regulatory pathways that underlie the identity and diversity of mouse tissue macrophages. Nat Immunol. 2012;13(11):1118-1128.

13. Rosas M, et al. The transcription factor Gata6 links tissue macrophage phenotype and proliferative renewal. Science. 2014;344(6184):645-648.

14. Okabe Y, Medzhitov R. Tissue-specific signals control reversible program of localization and functional polarization of macrophages. Cell. 2014;157(4):832-844.

15. Yona S, et al. Fate mapping reveals origins and dynamics of monocytes and tissue macrophages under homeostasis. Immunity 2013;38(1):79-91.

16. Stables MJ, et al. Transcriptomic analyses of murine resolution-phase macrophages. Blood. 2011;118(26):e192-208.

17. Newson J, et al. Resolution of acute inflammation bridges the gap between innate and adaptive immunity. Blood. 2014;124(11):1748-1764.

18. He JQ, Wiesmann C, van Lookeren Campagne M. A role of macrophage complement receptor CRIg in immune clearance and inflammation. Mol Immunol. 2008;45(16):4041-4047.

19. Vogt L, et al. VSIG4, a B7 family-related protein, is a negative regulator of T cell activation. J Clin Invest. 2006;116(10):28172826.

20. Helmy KY, Katschke KJ Jr. Gorgani NN, Kljavin NM, Elliott JM, Diehl L, Scales SJ, Ghilardi N, and van Lookeren Campagne M. CRIg: a macrophage complement receptor required for phagocytosis of circulating pathogens. Cell. 2006;124(5):915-927.

21. Gautier EL, Ivanov S, Lesnik P, Randolph GJ. Local apoptosis mediates clearance of macrophages from resolving inflammation in mice. Blood. 2013;122(15):2714-2722.

22. Gorgani NN, et al. Complement receptor of the Ig superfamily enhances complement-mediated phagocytosis in a subpopulation of tissue resident macrophages. J Immunol. 2008;181(11):7902-7908.

23. Anders S, Huber W. Differential expression analysis for sequence count data. Genome Biol. 2010;11(10):R106.

24. Robinson MD, McCarthy DJ, Smyth GK. edgeR: a Bioconductor package for differential expression analysis of digital gene expression data. Bioinformatics. 2010;26(1):139-140.

25. Anders S, Reyes A, Huber W. Detecting differential usage of exons from RNA-seq data. Genome Res. 2012;22(10):2008-2017.

26. O'Neill LA, Sheedy FJ, McCoy CE. MicroRNAs: the fine-tuners of Toll-like receptor signalling. Nat Rev Immunol. 2011;11(3):163-175

27. Godec J, et al. Compendium of immune signatures identifies conserved and species-specific biology in response to inflammation. Immunity. 2016;44(1):194-206.

28. Abbas AR, et al. Immune response in silico (IRIS): immune-specific genes identified from a compendium of microarray expression data. Genes Immun. 2005;6(4):319-331.

29. Segura E, et al. Human inflammatory dendritic cells induce Th17 cell differentiation. Immunity. 2013;38(2):336-348.

30. Huang dW, Sherman BT, Lempicki RA. Systematic and integrative analysis of large gene lists using DAVID bioinformatics resources. Nat Protoc. 2009;4(1):44-57.

31. Xue J, et al. Transcriptome-based network analysis reveals a spectrum model of human macrophage activation. Immunity. 2014;40(2):274-288

32. Jenkins SJ, et al. Local macrophage proliferation, rather than recruitment from the blood, is a signature of TH2 inflammation. Science. 2011;332(6035):1284-1288.

33. Gundra UM, et al. Alternatively activated macrophages derived from monocytes and tissue macrophages are phenotypically and functionally distinct. Blood. 2014;123(20):e110-e122. 
34. Bajaj JS, et al. Altered profile of human gut microbiome is associated with cirrhosis and its complications. $J$ Hepatol. 2014;60(5):940-947.

35. Leo MA, Lieber CS. Hepatic vitamin A depletion in alcoholic liver injury. N Engl J Med. 1982;307(10):597-601.

36. Epelman S, Lavine KJ, Randolph GJ. Origin and functions of tissue macrophages. Immunity. 2014;41(1):21-35.

37. Lamb J, et al. The Connectivity Map: using gene-expression signatures to connect small molecules, genes, and disease. Science. 2006;313(5795):1929-1935.

38. Davies LC, et al. Distinct bone marrow-derived and tissue-resident macrophage lineages proliferate at key stages during inflammation. Nat Commun. 2013;4:1886

39. Davies LC, Rosas M, Smith PJ, Fraser DJ, Jones SA, Taylor PR. A quantifiable proliferative burst of tissue macrophages restores homeostatic macrophage populations after acute inflammation. Eur J Immunol. 2011;41(8):2155-2164.

40. Kim KH, et al. CRIg signals induce anti-intracellular bacterial phagosome activity in a chloride intracellular channel 3-dependent manner. Eur J Immunol. 2013;43(3):667-678.

41. Katschke KJ Jr, et al. A novel inhibitor of the alternative pathway of complement reverses inflammation and bone destruction in experimental arthritis. J Exp Med. 2007;204(6):1319-1325.

42. Jung $\mathrm{K}$, et al. Protective role of V-set and immunoglobulin domain-containing 4 expressed on kupffer cells during immune-mediated liver injury by inducing tolerance of liver T- and natural killer T-cells. Hepatology. 2012;56(5):1838-1848.

43. Jung K, Seo SK, Choi I. Endogenous VSIG4 negatively regulates the helper T cell-mediated antibody response. Immunol Lett. 2015;165(2):78-83.

44. Qiao Q, et al. A novel CRIg-targeted complement inhibitor protects cells from complement damage. FASEB J. 2014;28(11):4986-4999.

45. Allen JE, Sutherland TE. Host protective roles of type 2 immunity: parasite killing and tissue repair, flip sides of the same coin. Semin Immunol. 2014;26(4):329-340.

46. Kim KE, et al. Role of $\mathrm{CD} 11 \mathrm{~b}^{+}$macrophages in intraperitoneal lipopolysaccharide-induced aberrant lymphangiogenesis and lymphatic function in the diaphragm. Am J Pathol. 2009;175(4):1733-1745.

47. Lee KM, Danuser R, Stein JV, Graham D, Nibbs RJ, Graham GJ. The chemokine receptors ACKR2 and CCR2 reciprocally regulate lymphatic vessel density. EMBO J. 2014;33(21):2564-2580.

48. Ma Y, et al. Protein kinase calpha regulates the expression of complement receptor Ig in human monocyte-derived macrophages. J Immunol. 2015;194(6):2855-2861.

49. Gorgani NN, et al. Regulation of CRIg expression and phagocytosis in human macrophages by arachidonate, dexamethasone, and cytokines. Am J Pathol. 2011;179(3):1310-1318

50. Guo S, et al. Down-regulation of Z39Ig on macrophages by IFN-gamma in patients with chronic HBV infection. Clin Immunol. 2010;136(2):282-291.

51. Jenkins SJ, et al. IL-4 directly signals tissue-resident macrophages to proliferate beyond homeostatic levels controlled by CSF-1. JExp Med. 2013;210(11):2477-2491.

52. Hume DA, MacDonald KP. Therapeutic applications of macrophage colony-stimulating factor-1 (CSF-1) and antagonists of CSF-1 receptor (CSF-1R) signaling. Blood. 2012;119(8):1810-1820.

53. Stutchfield BM, et al. CSF1 restores innate immunity after liver injury in mice and serum levels indicate outcomes of patients with acute liver failure. Gastroenterology. 2015;149(7):1896-1909.

54. Charlson ME, Pompei P, Ales KL, MacKenzie CR. A new method of classifying prognostic comorbidity in longitudinal studies: development and validation. J Chronic Dis. 1987;40(5):373-383.

55. Bokil NJ, et al. Intramacrophage survival of uropathogenic Escherichia coli: differences between diverse clinical isolates and between mouse and human macrophages. Immunobiology. 2011;216(11):1164-1171.

56. Schaale K, et al. Strain- and host species-specific inflammasome activation, IL-1 $\beta$ release, and cell death in macrophages infected with uropathogenic Escherichia coli. Mucosal Immunol. 2016;9(1):124-136.

57. Dobin A, et al. STAR: ultrafast universal RNA-seq aligner. Bioinformatics. 2013;29(1):15-21.

58. Li H, et al. The Sequence Alignment/Map format and SAMtools. Bioinformatics. 2009;25(16):2078-2079.

59. Anders S, Pyl PT, Huber W. HTSeq - a Python framework to work with high-throughput sequencing data. Bioinformatics. 2015;31(2):166-169

60. Love MI, Huber W, Anders S. Moderated estimation of fold change and dispersion for RNA-seq data with DESeq2. Genome Biol. 2014;15(12):550. 